\title{
Proceso de compras en las empresas de producción social de la industria petrolera venezolana
}

\author{
Purchasing process in social production companies in the Venezuelan \\ oil industry
}

Artículo recibido en abril 2019

Arbitrado en mayo 2019

Publicado en julio 2019

\section{Glenda Pérez}

glendaperez1244@gmail.com ORCID: 0000-0001-5503-7239

Industria Petrolera Venezolana - Venezuela

RESUMEN | El estudio buscó analizar el proceso de compras en las empresas de producción social prestadoras de servicio a la industria petrolera venezolana. Metodológicamente se tipificó como una investigación descriptiva, con un diseño no experimental, transversal de campo. La población quedó constituida por diez empresas de producción social que prestan servicios al programa de gerencia de PDVSA CVP. La técnica utilizada fue la encuesta y el instrumento un cuestionario con escala de frecuencia de cinco opciones de respuestas, compuesto por 15 ítems, se utilizó el juicio de expertos para la validez y el coeficiente Alfa Cronbach para su confiabilidad, obteniéndose un valor de 0,98. Las frecuencias relativas y la media aritmética se usaron para el análisis de los resultados. Se evidencio que estas empresas desarrollan las actividades básicas que permiten moderadamente detectar y describir sus necesidades, a fin de adquirir los productos y gestionar los servicios necesarios para el buen funcionamiento.

Palabras clave Keywords
Actividades básicas, necesidades, proceso de compras, producción social, productos, servicios

\begin{abstract}
The study sought to analyze the purchasing process in social production companies that provide services to the Venezuelan oil industry. Methodologically it was typified as a descriptive investigation, with a non-experimental, cross-sectional field design. The population was made up of ten social production companies that provide services to the PDVSA CVP management program. The technique used was the survey and the instrument was a questionnaire with a frequency scale of five response options, consisting of 15 items, expert judgment was used for validity and the Alpha Cronbach coefficient for its reliability, obtaining a value of 0,98 . Relative frequencies and arithmetic mean were used for the analysis of the results. It was evident that these companies carry out the basic activities that allow their needs to be detected and described in a modest way, in order to acquire the products and manage the services necessary for their proper functioning.
\end{abstract}

Basic activities, needs, purchasing process, social production, products, services 
INTRODUCCIÓN

El ser humano en la búsqueda continua de la satisfacción de sus necesidades, ha puesto en práctica distintas formas de intercambio $y$ abastecimiento que se han perfeccionado a través del tiempo hasta llegar a las prácticas comerciales que se desarrollan en la actualidad. Del mismo modo, las organizaciones o entidades sociales en las que varias personas interactúan con el propósito de alcanzar objetivos específicos, demandan constantemente una serie de recursos del entorno para incorporarlos a sus procesos productivos.

En este sentido, Heredia (2007), señala que las compras o adquisiciones se tornan en el elemento fundamental para el desarrollo productivo, ya que se convierten en la fuente de abastecimiento, que alimenta y soporta el engranaje competitivo de los diferentes productos que más tarde se ofertarán en el mercado.

Refiere el citado autor, que dada la importancia de las compras y su estrecha relación con los procesos productivos, la gestión de compras toma como base la planificación, la ejecución y el control o seguimiento, de todas y cada una de las acciones relacionadas directa o indirectamente con las adquisiciones en la empresa, es decir, no sólo se ocupa de un simple intercambio comercial.

En efecto, según Martínez (2007), el fin concreto de la gestión de compras consiste en planificar el suministro de los materiales, bajo las mejores condiciones posibles a los distintos sectores de la empresa; con las características mínimas necesarias requeridas, es decir: precio, calidad, condiciones de entrega, además de las condiciones de pago; que son necesarios para alcanzar los objetivos que la administración de la misma ha definido, en la que se deben cumplir la requisición y solicitud de materiales, orden de compra o pedido, recepción y comprobación de materiales.

De acuerdo a lo anterior, a juicio de la investigadora, las compras involucran procesos complejos los cuales bajo un buen seguimiento y control de los pasos que debe cumplir, garantizaran gran parte de los objetivos de la empresa, disminuyendo costos que se reflejan en el incremento de las utilidades de la misma. Estos procesos no son iguales o fijos para todas las empresas, ello va a depender de su razón social (actividad económica) y por supuesto a sus políticas internas.

Dentro de este marco de requerimientos y retos continuos, están inmersas las llamadas empresas de producción social (EPS), las cuales en Venezuela surgen como instrumento para organizar y realizar un proceso de integración entre el proceso productivo y la acción de la comunidad, lo cual ayudará el desarrollo de estas, generando mejores condiciones de vida dentro de las comunidades venezolanas.

Desde esta perspectiva, se pueden definir las EPS según Vásquez (2008), como entidades económicas dedicadas a la producción de bienes, obras y servicios, en las cuales el trabajo tiene significado propio, no alienado y auténtico. No existe discriminación social en el trabajo y de ningún tipo de trabajo, no existen privilegios asociados a la posición jerárquica, con igualdad sustantiva entre sus integrantes, basadas en una planificación participativa y protagónica y bajo régimen de propiedad estatal, colectiva o la combinación de ambas. 
Como parte de este marco de acción, el gobierno venezolano ha venido fomentado este tipo de organizaciones, a través de la Constitución de la República Bolivariana de Venezuela del año 1999, en el proyecto político nacional (CRBV) y las Líneas Generales del Plan de Desarrollo Económico y social (PNSB).

En el caso de la industria estatal como Petróleos de Venezuela S.A (PDVSA), tal como lo reseña en su página oficial de internet (2019), el programa de Empresas de Producción Social (EPS), surge en el marco del plan Siembra Petrolera 2005-2030, como parte del nuevo modelo económico y social del país, donde se hace imperativo implantar y desarrollar un programa extraordinario que impulse en el seno de la industria, la democratización de las oportunidades apalancadas en la demanda de bienes, ejecución de obras y contratación de servicios que posee la contratación, lo cual consolidaría la construcción de la nueva PDVSA como un modelo inédito de relaciones con las empresas y las comunidad.

En atención a esto, el presente estudio, se enfocará en este tipo de organizaciones, que privilegia el trabajo en equipos auto-dirigidos, según se estipula en los estatutos nacionales, y que actualmente se ha fomentado de modo considerable ya que tienen un papel fundamental en el llamado realizado por el ejecutivo nacional, en referencia a impulsar el modelo productivo socialista, a través de la prestación de sus servicios a la industria petrolera del país. Esto lo confirma la Ley Orgánica de Hidrocarburos (2001), al establecer que los ingresos que en razón de los hidrocarburos reciba la Nación estarán destinados a financiar la salud, la educación, la formación de fondos de estabilización macroeconómica y la inversión productiva, de manera que se logre una apropiada vinculación del petróleo con la economía nacional, todo ello en función del bienestar del pueblo.

Surge así el Programa de Gerencia Petrolera para la Capacitación de Empresas de Producción Social (EPS) de PDVSA CVP, a lo largo y ancho de Venezuela y constituidas por profesionales universitarios, como consecuencia de la necesidad de transformar las relaciones de producción y de negocios, que tradicionalmente existían con las empresas contratistas proveedoras de bienes muebles, obras y servicios comerciales, o empresas de consultoría prestadoras de servicios profesionales, del sector de los hidrocarburos, a fin de propiciar e incentivar un cambio de actitud del empresariado del sector privado nacional, para que oriente sus acciones en función de cumplir con el compromiso de responsabilidad social que tiene con el país y las comunidades que las integran, dentro o fuera de su entorno, con el propósito de satisfacer sus necesidades inmediatas y perentorias, contribuyendo con su desarrollo futuro y sostenido (PDVSA, página oficial de internet 2019).

De manera que, al enmarcar la gestión de compras en las EPS prestadoras de servicio al programa de gerencia de PDVSA CVP, es importante considerar que por ser estas de reciente creación no se han consolidado los departamentos de compras. Sobre este particular, es de hacer notar que las entrevistas diagnósticas no estructuradas, la observación directa por parte de la investigadora, así como la revisión de algunos trabajos realizados en este ámbito permiten revelar que en su mayoría las 
EPS afrontan problemas para llevar a cabo de manera eficaz y eficiente su gestión de compras, debido a: Retrasos en los procesos de compras, por duplicidad de funciones, y niveles de aprobación que afectan el alcance en la adquisición de bienes y de servicios, lo cual coloca en riesgo la aceptación y la plena satisfacción del cliente, y por ende, la concreción en el incremento de las operaciones de producción.

En consecuencia, el proceso de compra, requiere ser estudiado, de forma tal, de contribuir a la mejora continua, eficiencia y eficacia de la gestión. De acuerdo a esto, surge la necesidad de llevar a cabo esta investigación acerca de analizar el proceso de compras en las empresas de producción social prestadoras de servicio a la industria petrolera venezolana.

\section{Proceso de compras}

La gestión de compras es la vía que facilita el mejoramiento de los procesos organizacionales, cubriendo las necesidades de las empresas de producción social de manera estratégica con elementos externos a ella, siendo esto un proceso de planificación, organización, dirección y control de actividades relacionadas a compras.

Para Heredia (2007) es un elemento fundamental para el desarrollo del proceso productivo, ya que se convierten en la fuente de abastecimiento, que finalmente alimenta $y$ soporta el engranaje competitivo de los diferentes productos que más tarde se ofertarán en el mercado; por lo cual requiere de ciertas etapas que van desde la detección y descripción de la necesidad hasta el seguimiento y control de compras, apoyándose en las plataformas tecnológicas, así como en los mecanismos de evaluación.

Ello evidencia que la gestión de compras, se fundamenta en los procesos productivos medulares de las empresas, lo que aumenta el grado de credibilidad y competitividad ante los competidores, y que para ello se debe deben propiciar el aprovechamiento de las plataformas tecnológicas como tecnologías de información que apoyen la gestión institucional mediante el manejo apropiado de la información y la implementación de soluciones ágiles y de amplio alcance. Además de disponer de mecanismos de control que les permita revisar lo que ha sucedido y decidir cómo tuvo éxito y cómo no lo tuvo éxito.

En este orden de ideas, para Kotler y Armstrong (2008) el proceso de compra se inicia mucho antes de la compra propiamente dicha, continúa durante mucho tiempo después y consta de cinco etapas fundamentales: reconocimiento de la necesidad, búsqueda de información, evaluación de alternativas, decisión de compra y comportamiento posterior a la compra. Normalmente, los consumidores pasan por las cinco etapas en cada compra, pero en compras de rutina los consumidores a menudo se saltan algunas de esas etapas, o invierten su orden.

Para Hawkins y otros (2004) es un proceso mediante el cual una persona evalúa detenidamente los atributos de un producto, marca o servicio y que selecciona, de manera racional, el que soluciona la necesidad reconocida al menor costo. Existe una manera racional y funcional para tomar decisiones, pero algunas otras implican poco esfuerzo consciente. 
En otras palabras, Arellano (2002) en su última edición, plantea que cada acción de los individuos está íntimamente relacionada con el resultado de la compra, aunque, en realidad el proceso es más complicado, es decir, no se toma una sola decisión en el momento de la compra, sino que esta se conforma por diversas decisiones intermedias.

A criterio de la investigadora, el proceso de compras es el encargado de adquirir los productos y gestionar los servicios necesarios para facilitar el mejoramiento de los procesos organizacionales, cubriendo las necesidades de las empresas de producción social de manera estratégica con elementos externos a ella, siendo esto un proceso de planificación, organización, dirección y control de actividades. Para ello se requiere la detección y descripción de la necesidad, investigación de fuentes de aprovisionamiento, preparación de la compra, ejecución de la compra y seguimiento y control.

\section{Detección y descripción de la necesidad}

Para Martínez (2007) consiste en la descripción detallada material de la necesidad (qué), incluyendo la previsión cantidad y para cuando. El departamento de compras debe participar desde el principio, evaluando el coste de los materiales, proponiendo componentes que existan en el mercado, encargando desarrollos parciales a proveedores e informando a la oficina de proyectos de nuevos materiales y técnicas.

En este orden de ideas, Montoya (2002), plantea que el comprador identifica necesidades a través de la información obtenida de los almacenes o de los usuarios de la empresa, la promesa de servicio a los clientes o usuarios, las investigaciones con los clientes internos o externos, el análisis de lo que ofrece o hace la competencia, las visitas a ferias o exposiciones, entre otros.

Resalta el autor, que el comprador siempre deberá conocer con exactitud cuál es la actividad de la empresa para la cual trabaja, la vocación del surtido o el tipo de productos que debe adquirir, el objetivo de posicionamiento o el servicio que quiere prestar, la imagen que quiere proyectar o el usuario que debe atender, la calidad que debe ofrecer, la capacidad de pago que tenga para cumplir, la cantidad que tiene en su inventario, la cantidad que demanda o el consumo en un período de tiempo determinado, los pedidos que tiene por recibir, la disponibilidad de espacio o la necesidad de almacenar o exhibir la mercancía, las facilidades para custodiar los productos de alto riesgo, la posibilidad de buscar sustitutos, el precio y la calidad que está dispuesto a pagar.

De acuerdo con Kotler y Armstrong (2008) la detección y descripción de las necesidades es el punto de partida para el proceso de decisión de compra. La necesidad puede ser provocada por estímulos internos cuando la necesidad normal de una persona se intensifica al punto de convertirse en un impulso. También puede ser provocada por estímulos externos.

El reconocimiento del problema viene siendo la primera etapa del proceso de decisión del consumidor según Hawkins y otros (2004). Es la derivación de la discordancia entre un estado deseado y un estado real que es suficiente razón para activar o iniciar el proceso de decisión. Sin el reconocimiento de un problema no existe la necesidad de tomar decisión alguna.

Por otro lado, Arellano (2002) acota que en la etapa de reconocimiento del problema el sujeto 
reconoce la existencia de una carencia y acepta que debe realizar un esfuerzo para satisfacerla. En esta etapa también se realiza un análisis de los diversos medios para satisfacer la necesidad.

A efectos de esta investigación, para la investigadora en esta etapa se trata de determinar las necesidades que presenta la empresa o el departamento solicitante a través de la especificación técnica recogida en un documento en el que se describen de forma detallada y estandarizada los requerimientos mínimos para la creación, documentación, adquisición, uso y evaluación del producto/servicio que cubrirá dichas necesidades.

\section{Investigación de fuentes de aprovisionamiento}

Según Kotler y Armstrong (2008) la búsqueda de información dependerá de la intensidad de su impulso, de la cantidad de información inicial, de la facilidad para obtenerla, del valor que tenga para la persona la información adicional y de la satisfacción obtenida de la búsqueda. Los consumidores pueden obtener información a partir de fuentes personales, comerciales, públicas y empíricas (manejo, evaluación, uso del producto). Generalmente, de donde más se recibe información de un producto es de fuentes comerciales, pero sin embargo, las más eficaces suelen ser las personales. Conforme se obtiene más información, aumenta la conciencia y el conocimiento del consumidor.

La búsqueda de información según Hawkins y otros (2004) son procesos continuos y no pasivos que exigen actividades mentales y físicas. La información pertinente de la memoria de largo plazo sirve para determinar si se conoce una solución satisfactoria, cuales son las características de las posibles soluciones, las formas más adecuadas para comparar las soluciones, entre otros, a esto los referidos autores le llaman búsqueda interna. De no llegarse a una solución se inicia la búsqueda externa.

Por su parte, Arellano (2002), plantea que luego de aceptar el problema, se procede a buscar información sobre el mismo, comenzando por la información interna y consecuentemente (de ser necesario) por la información externa. La información interna se basa en la experiencia adquirida con la misma necesidad o con necesidades similares y en la manera en cómo se solucionó el problema anteriormente. La información externa se basa en el medio comercial.

Desde estas perspectivas, la investigadora considera que se trata pues de investigar y analizar los diversos tipos de productos/servicios que cubren la necesidad junto con sus proveedores para identificar el modelo más óptimo al mejor precio.

\section{Preparación de la compra}

Luego de realizado el estudio de mercado Martínez (2007) agrega que se debe acometer la eficaz prospección y examen de los posibles proveedores, para asegurar que tienen las condiciones necesarias y suficientes para satisfacer plenamente nuestras necesidades: hay que visitar sus talleres, almacenes, servicios técnicos, proceder a la recogida de informes acerca de su situación laboral, financiera, servicio a clientes. Estableciendo primeros contactos para conocerlos y decidir si pueden ser proveedores potenciales de nuestra empresa. Además, 
debemos poder esbozar las bases de una futura política de compras.

Por su parte Montoya (2002) agrega que cada empresa, dependiendo de su tamaño, complejidad, políticas y procedimientos internos, exige la documentación necesaria para oficializar la compra, recibir los pedidos, legalizar y cancelar las facturas.

En otro orden de ideas, Kotler y Armstrong (2008) plantean que la evaluación de alternativas es la manera en que el consumidor procesa su información para efectuar su decisión. Los consumidores no siguen un solo proceso de evaluación para resolver todas las situaciones de compra. El consumidor adquiere actitudes hacia las diferentes marcas a través de un proceso de evaluación. La forma en que se evalúan las alternativas de compra depende del consumidor individual y de la situación de compra especifica. En algunos casos se realizan cálculos y se razona lógicamente; en otros los mismos consumidores casi no evalúan (actúan por intuición).

Arellano (2002) plantea que al final de la búsqueda, el individuo estudiara los datos y tomara una decisión donde se implicaran varios elementos tales como la cantidad a gastar, las características generales del producto y el esfuerzo en el acto de compra. Es importante tomar en cuenta aspectos como las actitudes que tiene la persona frente a un producto o tienda, expectativas de ingreso, entre otros, es decir, a todo aquello que pueda generar una inclinación sobre lo que va a comprar.

Para la investigadora en esta fase del proceso de compra, una vez definida la estrategia de compras que seguirá la empresa es el momento de establecer unas pautas para preparar las posibles negociaciones. Antes de negociar deberá tener claro cuál es el objetivo, la argumentación para conseguirlo y la información que dará soporte al argumento.

\section{Ejecución de la compra}

Martínez (2007) indica que en esta fase del proceso de compras se inicia con la petición de ofertas que deberán cumplir con las condiciones fundamentales de la política de compras. Incluye el análisis y evaluación de las ofertas de acuerdo a los criterios establecidos en la etapa de preparación de la compra, por lo que se debe comparar no solamente el precio, sino también entrar en la valoración de factores como la garantía de cumplimiento de especificaciones técnicas, la fiabilidad de los plazos de entrega, la posible continuidad en el suministro, entre otros; repartiendo luego el pedido entre varios proveedores para proteger a la empresa de circunstancias imprevistas.

De acuerdo a lo planteado por Montoya (2002) es imperante precisar la necesidad de establecer la mayor claridad posible en los términos acordados en una negociación, y evitar futuros reclamos por interpretaciones 0 suposiciones que se puedan presentar después de concretar una compra, hace que el comprador deba definir una serie de condiciones que se deben registrar en la orden de compra, para que en el momento de recibir el pedido, el proveedor pueda conocer las condiciones y reglas que tiene establecido el cliente para el recibo y posterior cancelación de la factura y proceda a informar al administrador de las compras sobre los términos que se deben cambiar o no se pueden cumplir.

Se puede afirmar entonces que dentro del proceso de compras en las empresas de producción social prestadoras de servicio al 
programa de gerencia petrolera de PDVSA CVP, se establecen obligaciones inviolables plasmadas en documento, de manera clara y con fundamentos en la ley y las políticas tanto de quien compra como de quien vende; de tal manera de que no dar pie a las mala interpretaciones que puedan generar incumplimiento en lo acordado y esto va desde la cantidad solicitada hasta la calidad, cantidad, precios, forma de pagos, entre otros.

Por otra parte, se busca lo que Kotler y Armstrong (2008) señala en la decisión de compra, que es adquirir la marca de preferencia, al respecto existen dos factores que pueden interponerse entre la intención de compra y la decisión de compra. El primer factor viene dado por las actitudes de otros, es decir, si alguien que el consumidor considere importante emita una opinión negativa sobre el producto que se va a adquirir, las posibilidades de compra se verán reducidas.

El segundo factor está compuesto por factores de situación inesperados en donde el consumidor puede formar una intención de compra en base a unos factores supuestos (ingresos, beneficios que espera obtener del producto, entre otros) y estos procesos inesperados podrían alterar la intención de compra. Es así, como las preferencias e incluso las intenciones de compra no siempre aseguran una compra real.

A partir de las consideraciones expuesta, para la investigadora la ejecución de la compra es la etapa que permite la adquisición de materia prima o insumos externos a la empresa y que son clave para el funcionamiento productivo de la organización, garantizando entonces el cumplimiento de las solicitudes realizadas por los clientes.

\section{Seguimiento y control}

Kotler y Armstrong (2008) plantean que la tarea del especialista en mercadeo no termina con la compra del producto ya que después de adquirido el mismo, el comprador puede quedar satisfecho o no y presentara un comportamiento posterior a la compra la cual es de sumo interés para la empresa. Lo que determina si un consumidor ha quedado insatisfecho o no es la relación que hay entre las expectativas del consumidor y el desempeño percibido del producto. Si el producto no cumple el consumidor se decepciona, si cumple quedará satisfecho y si excede las expectativas quedará encantado.

Para Arellano (2002) en la utilización y análisis post-compra el individuo inicia inmediatamente un proceso de análisis de la calidad de su compra. En esta etapa, el individuo es más atento a cualquier información relacionada con el producto, es decir, atento a precios, características de los productos, entre otras cosas. En este orden de ideas, el consumidor puede perturbarse si luego de realizada la compra encuentra algo mejor, lo cual podría llevarlo a un descontento inicial.

En el mismo orden de ideas, Martínez (2007) contempla que con la firma del contrato o con el hecho de pasar un pedido no termina la responsabilidad de compras. Además de cursar las órdenes de entrega hay que realizar un cuidadoso seguimiento, que habitualmente debe incluir no sólo el control administrativo sino también visitas a los proveedores; con el doble 
objetivo de tener la seguridad del cumplimiento de los plazos o para detectar a tiempo los posibles retrasos y poder adoptar las medidas correctoras.

Montoya (2002), agrega a lo anterior que cada empresa, dependiendo de su tamaño, complejidad, políticas y procedimientos internos, exige la documentación necesaria para oficializar la compra, recibir los pedidos, legalizar y cancelar las facturas.

Cabe destacar que en Venezuela además de las políticas y procedimientos internos citados en el párrafo anterior, las organizaciones del sector público, para realizar las compras, deben regirse por las disposiciones contempladas la Ley de Contrataciones Públicas (2009), en la que precisamente se establece en relación a la etapa de preparación de la compra, que las reglas, condiciones y criterios aplicables a cada contratación o compra deben ser objetivos, de posible verificación y revisión, y se establecerán en un documento denominado pliego de condiciones.

En esta etapa, a criterio de la investigadora, se lleva un gran peso en cuanto a repercusiones que pueda crear el cliente hacia las empresas de producción social prestadoras de servicio al programa de gerencia petrolera de PDVSA CVP, y que dependiendo de cómo se aborden las sugerencias y problemas de los clientes se podrá fidelizar y crear un mejor lazo con el mismo.

\section{MATERIALES Y MÉTODO}

Metodológicamente se tipificó como una investigación de tipo descriptivo, con un diseño no experimental, transeccional descriptivo y de campo. La población quedó constituida por diez empresas de producción social que prestan servicios al programa de gerencia de PDVSA CVP ubicadas en el oriente y occidente del país, siendo las unidades informantes diez (10) gerentes y diez (10) coordinadores de administración.

La técnica de recolección de datos utilizada fue la encuesta y el instrumento un cuestionario con escala de frecuencia de cinco (05) opciones de respuestas que van desde Siempre(S), Casi Siempre (CS), Algunas Veces (AV), Casi Nunca (CN) y Nunca(N), compuesto por 15 ítems, se utilizó el juicio de expertos para la validez del mismo y el coeficiente Alfa Cronbach para su confiabilidad, obteniéndose un valor de 0,98 indicando que el instrumento posee muy alta confiabilidad.

El análisis estadístico de la información, se realizó a través del uso de la estadística descriptiva por medio de las frecuencias relativas; así como de la técnica de medidas de tendencia central. Partiendo de estas premisas se realizó un baremo con la finalidad de analizar e interpretar la media aritmética derivada de someter a la estadística descriptiva los datos obtenidos de la aplicación del cuestionario. El mismo se diseñó considerando los puntajes máximos asignados a cada reactivo, además de los atributos establecidos, tal como se muestra en el cuadro 1. 
Cuadro 1. Categoría de análisis para la interpretación del promedio

\begin{tabular}{ccc}
\hline Dimensión & $\begin{array}{c}\text { Rango para } \\
\text { la media }\end{array}$ & Nivel de respuesta para la dimensión y sus indicadores \\
\hline & $4.20-5.00$ & Muy alta presencia \\
& $3.40-4.20$ & Alta presencia \\
Proceso de compras & $2.60-3.40$ & Moderada presencia \\
& $1.80-2.60$ & Baja presencia \\
& $1.00-1.80$ & No hay presencia \\
\hline
\end{tabular}

Fuente: Elaboración propia (2019)

De igual manera, se asumió que la suma de las frecuencias relativas de las alternativas (siempre y casi siempre) para los indicadores cuando supera el $75 \%$ se considera como tendencia positiva en el proceso de compras en las empresas de producción social prestadoras de servicio al programa de gerencia petrolera de PDVSA CVP, tal como se aprecia en el cuadro 2.

Cuadro 2. Categoría de análisis para la frecuencia relativa

\begin{tabular}{cc}
\hline Alternativa & Tendencia \\
\hline $\mathrm{S}+\mathrm{CS} \geq 66 \%$ & La tendencia se considera positiva \\
$33 \leq \mathrm{S}+\mathrm{CS}<66 \%$ & La tendencia se considera neutral \\
$\mathrm{S}+\mathrm{CS}<33 \%$ & La tendencia se considera negativa \\
\hline
\end{tabular}

Fuente: Elaboración propia (2019)

\section{RESULTADOS Y DISCUSION}

Se presenta a continuación los resultados obtenidos para cada uno de los indicadores que conforman el objetivo dirigido a analizar el proceso de compras en las empresas de producción social prestadoras de servicio a la industria petrolera venezolana, dando inicio al análisis a través del indicador detección y descripción de la necesidad, cuyos valores se reflejan en la tabla 1 evidenciándose en cada uno de los ítems aplicados, valores agrupados en las opciones siempre y casi siempre que alcanzan solo el $35 \%$ marcando tendencia neutral.

Aunado a esto, los ítems se posicionaron en la categoría de moderada presencia $(2,93)$ afirmando los encuestados que identifican necesidades a través de requerimientos de los usuarios, identifican necesidades a través de la información obtenida de los almacenes y realizan un análisis de los diversos medios para satisfacer la necesidad. 
Tabla 1. Indicador: Detección y descripción de la necesidad

\begin{tabular}{|c|c|c|c|c|c|c|}
\hline Ítemes & \multicolumn{2}{|c|}{$\begin{array}{l}\text { Se identifican } \\
\text { necesidades a través } \\
\text { de requerimientos de } \\
\text { los usuarios. }\end{array}$} & \multicolumn{2}{|c|}{$\begin{array}{l}\text { Se identifican } \\
\text { necesidades a través de } \\
\text { la información obtenida } \\
\text { de los almacenes }\end{array}$} & \multicolumn{2}{|c|}{$\begin{array}{l}\text { Se realiza un análisis de los } \\
\text { diversos medios para } \\
\text { satisfacer la necesidad. }\end{array}$} \\
\hline Alternativas & $\mathrm{Fa}$ & $\mathrm{Ha}$ & $\mathrm{Fa}$ & $\mathrm{Ha}$ & $\mathrm{Fa}$ & $\mathrm{Ha}$ \\
\hline Siempre(S) & 0 & 0 & 3 & 15 & 3 & 15 \\
\hline Casi Siempre (CS) & 7 & 35 & 4 & 20 & 4 & 20 \\
\hline Algunas Veces (AV) & 7 & 35 & 3 & 15 & 7 & 35 \\
\hline Casi Nunca (CN) & 3 & 15 & 7 & 35 & 3 & 15 \\
\hline $\operatorname{Nunca(N)}$ & 3 & 15 & 3 & 15 & 3 & 15 \\
\hline Total & 20 & 100 & 20 & 100 & 20 & 100 \\
\hline Alternativa & \multicolumn{2}{|c|}{$33 \leq S+C S<66 \%$} & \multicolumn{2}{|c|}{$33 \leq S+C S<66 \%$} & \multicolumn{2}{|c|}{$33 \leq S+C S<66 \%$} \\
\hline Tendencia & \multicolumn{2}{|c|}{ Neutral } & \multicolumn{2}{|c|}{ Neutral } & \multicolumn{2}{|c|}{ Neutral } \\
\hline Promedio/Ítemes & \multicolumn{2}{|c|}{2,90} & \multicolumn{2}{|c|}{2,85} & \multicolumn{2}{|c|}{3,05} \\
\hline Categoría & \multicolumn{2}{|c|}{ Moderada presencia } & \multicolumn{2}{|c|}{ Moderada presencia } & \multicolumn{2}{|c|}{ Moderada presencia } \\
\hline Promedio/Indicador & \multicolumn{6}{|c|}{2,93} \\
\hline Categoría & \multicolumn{6}{|c|}{ Moderada presencia } \\
\hline
\end{tabular}

Fuente. Elaboración propia (2019)

Según los hallazgos encontrados, la investigadora infiere que las empresas de producción social prestadoras de senvicio al programa de gerencia de PDVSA CVP, moderadamente realizan una descripción detallada del material que necesitan, a través de la información obtenida de los almacenes o de los usuarios de la empresa, a fin de lograr cumplir con las exigencias del mercado donde están inmersas.

Logrando una moderada congruencia con la teoría expuesta por Kotler y Armstrong (2008), quienes afirman que la detección y descripción de las necesidades es el punto de partida para el proceso de decisión de compra, esta necesidad puede ser provocada por estímulos internos cuando la necesidad normal de una persona se intensifica al punto de convertirse en un impulso. También puede ser provocada por estímulos externos.

En cuanto al indicador investigación de fuentes de aprovisionamiento, los resultados revelan valores agrupados en las opciones siempre y casi siempre que alcanzan el 35\% para los ítems 4 y 5, marcando tendencia neutral, no obstante, para el ítem 6 se encuentra en las opciones siempre y casi siempre con un acumulado del $50 \%$ demostrando también una tendencia neutral, tal como puede observarse en la tabla 2.

En este sentido, los sujetos informantes afirmaron con moderada presencia que se realizan investigaciones sobre las características del mercado de producción específico del 
producto a comprar, realizan revisiones del consumo histórico del material antes de realizar las compras e identifican con anticipación a la compra, cuales son los posibles proveedores que pueden vender el producto requerido.

Tabla 2. Indicador: Investigación de fuentes de aprovisionamiento

\begin{tabular}{|c|c|c|c|c|c|c|}
\hline \multirow{2}{*}{$\begin{array}{l}\text { Ítemes } \\
\text { Alternativas } \\
\end{array}$} & \multicolumn{2}{|c|}{$\begin{array}{l}\text { Se realizan } \\
\text { investigaciones sobre } \\
\text { las características del } \\
\text { mercado de } \\
\text { producción específico } \\
\text { del producto a } \\
\text { comprar }\end{array}$} & \multicolumn{2}{|c|}{$\begin{array}{l}\text { Antes de realizar las } \\
\text { compras, se realizan } \\
\text { revisiones del consumo } \\
\text { histórico del material. }\end{array}$} & \multicolumn{2}{|c|}{$\begin{array}{l}\text { Se identifican con } \\
\text { anticipación a la compra } \\
\text { cuales son los posibles } \\
\text { proveedores que pueder } \\
\text { vender el producto } \\
\text { requerido }\end{array}$} \\
\hline & $\mathrm{Fa}$ & На & $\mathrm{Fa}$ & На & $\mathrm{Fa}$ & $\mathrm{Ha}$ \\
\hline Siempre(S) & 3 & 15 & 4 & 20 & 4 & 20 \\
\hline Casi Siempre (CS) & 4 & 20 & 3 & 15 & 6 & 30 \\
\hline Algunas Veces (AV) & 7 & 35 & 0 & 0 & 3 & 15 \\
\hline Casi Nunca (CN) & 3 & 15 & 10 & 50 & 7 & 35 \\
\hline Nunca(N) & 3 & 15 & 3 & 15 & 0 & 0 \\
\hline Total & 20 & 100 & 20 & 100 & 20 & 100 \\
\hline Alternativa & \multicolumn{2}{|c|}{$33 \leq S+C S<66 \%$} & \multicolumn{2}{|c|}{$33 \leq S+C S<66 \%$} & \multicolumn{2}{|c|}{$33 \leq S+C S<66 \%$} \\
\hline Tendencia & \multicolumn{2}{|c|}{ Neutral } & \multicolumn{2}{|c|}{ Neutral } & \multicolumn{2}{|c|}{ Neutral } \\
\hline Promedio/Ítemes & \multicolumn{2}{|c|}{3,05} & \multicolumn{2}{|c|}{2,75} & \multicolumn{2}{|c|}{3,35} \\
\hline Categoría & \multicolumn{2}{|c|}{ Moderada presencia } & \multicolumn{2}{|c|}{ Moderada presencia } & \multicolumn{2}{|c|}{ Moderada presencia } \\
\hline Promedio/Indicador & \multicolumn{6}{|c|}{3,05} \\
\hline Categoría & \multicolumn{6}{|c|}{ Moderada presencia } \\
\hline
\end{tabular}

Fuente. Elaboración propia (2019)

Estos resultados, a juicio de la investigadora, revelan que las empresas bajo estudio, moderadamente reúnen y analizan la información necesaria de todos los artículos y materiales que se requieren; a través de la búsqueda y selección de proveedores, dando así validez a los postulados Kotler y Armstrong (2008), quienes refieren que la investigación de fuentes de aprovisionamiento requieren de fuentes de información y que esta dependerá de la intensidad de su impulso, de la cantidad de información inicial, de la facilidad para obtenerla, del valor que tenga para la persona la información adicional y de la satisfacción obtenida de la búsqueda.

Seguidamente, en la tabla 3, se resume el comportamiento para el indicador preparación de la compra, donde se observa que alcanzó frecuencias de $70 \%$, $70 \%$ y $35 \%$, agrupando las opciones siempre y casi siempre, indicando tendencia positiva de las respuestas obtenidas para los ítems 7 y 8, no así para el ítem 9 el cual revela una tendencia neutral. 
Al mismo tiempo, se muestra en promedio una media de 3,67 ubicándola en la categoría de alta presencia, como resultado de considerar los encuestados que con alta presencia se define el mecanismo de compra a emplear de acuerdo a la Ley de contrataciones y se define el mecanismo de compra a emplear de acuerdo a las políticas internas, pero con moderada presencia se establecen los criterios a exigir a los proveedores en el pliego de condiciones.

Tabla 3. Indicador: Preparación de la compra

\begin{tabular}{|c|c|c|c|c|c|c|}
\hline Ítemes & \multicolumn{2}{|c|}{$\begin{array}{l}\text { Define el mecanismo } \\
\text { de compra a emplear } \\
\text { de acuerdo a la Ley de } \\
\text { contrataciones }\end{array}$} & \multicolumn{2}{|c|}{$\begin{array}{l}\text { Define el mecanismo de } \\
\text { compra a emplear de } \\
\text { acuerdo a las políticas } \\
\text { internas }\end{array}$} & \multicolumn{2}{|c|}{$\begin{array}{l}\text { Se establecen los criterios } \\
\text { a exigir a los proveedores } \\
\text { en el pliego de condiciones }\end{array}$} \\
\hline Alternativas & $\mathrm{Fa}$ & На & $\mathrm{Fa}$ & $\mathrm{Ha}$ & $\mathrm{Fa}$ & На \\
\hline Siempre(S) & 7 & 35 & 7 & 35 & 3 & 15 \\
\hline Casi Siempre (CS) & 7 & 35 & 7 & 35 & 4 & 20 \\
\hline Algunas Veces (AV) & 3 & 15 & 6 & 30 & 10 & 50 \\
\hline Casi Nunca (CN) & 0 & 0 & 0 & 0 & 0 & 0 \\
\hline Nunca(N) & 3 & 15 & 0 & 0 & 3 & 15 \\
\hline Total & 20 & 100 & 20 & 100 & 20 & 100 \\
\hline Alternativa & \multicolumn{2}{|c|}{$S+C S \geq 66 \%$} & \multicolumn{2}{|c|}{$S+C S \geq 66 \%$} & \multicolumn{2}{|c|}{$33 \leq \mathrm{S}+\mathrm{CS}<66 \%$} \\
\hline Tendencia & \multicolumn{2}{|c|}{ Positiva } & \multicolumn{2}{|c|}{ Positiva } & \multicolumn{2}{|c|}{ Neutral } \\
\hline Promedio/Ítemes & \multicolumn{2}{|c|}{3,75} & \multicolumn{2}{|c|}{4,05} & \multicolumn{2}{|c|}{3,20} \\
\hline Categoría & \multicolumn{2}{|c|}{ Alta presencia } & \multicolumn{2}{|c|}{ Alta presencia } & \multicolumn{2}{|c|}{ Moderada presencia } \\
\hline Promedio/Indicador & \multicolumn{6}{|c|}{3,67} \\
\hline Categoría & \multicolumn{6}{|c|}{ Alta presencia } \\
\hline
\end{tabular}

Fuente. Elaboración propia (2019)

En atención a esto, a juicio de la investigadora las empresas de producción social prestadoras de servicio al programa de gerencia petrolera de PDVSA CVP, exigen la documentación necesaria para oficializar la compra, recibir los pedidos, legalizar y cancelar las facturas. Validando así, los postulados de Martínez (2006), quien expresa que en esta etapa se debe evaluar las condiciones necesarias y suficientes para satisfacer plenamente las necesidades, acorde a las normativas establecidas.
En lo concerniente al indicador ejecución de la compra, los resultados obtenidos se recogen en la tabla 4, donde se aprecia una concentración de respuestas en las opciones siempre y casi siempre, de 35\%,70\%, y 70\% respectivamente, marcando tendencia neutral para el ítem 10 y positiva para los ítems 11 y 12. Al mismo tiempo se muestra una media de 3,50 para este indicador, ubicándolo en la categoría de alta presencia.

Al detallar los resultados por ítems, se evidencia moderada presencia al considerar los 
encuestados que el análisis de las ofertas recibidas se realiza considerando los criterios previamente establecidos en el pliego de condiciones, al mismo tiempo con alta presencia afirmaron los encuestados que evalúan las ofertas de acuerdo a los criterios establecidos en la etapa de preparación de la compra y evalúan la fiabilidad de los plazos de entrega.

Tabla 4. Indicador: Ejecución de la compra

\begin{tabular}{|c|c|c|c|c|c|c|}
\hline \multirow{2}{*}{$\begin{array}{l}\text { Ítemes } \\
\text { Alternativas } \\
\end{array}$} & \multicolumn{2}{|c|}{$\begin{array}{l}\text { El análisis de las ofertas } \\
\text { recibidas se realiza } \\
\text { considerando los } \\
\text { criterios previamente } \\
\text { establecidos en el pliego } \\
\text { de condiciones }\end{array}$} & \multicolumn{2}{|c|}{$\begin{array}{l}\text { Se evalúan las ofertas de } \\
\text { acuerdo a los criterios } \\
\text { establecidos en la etapa } \\
\text { de preparación de la } \\
\text { compra }\end{array}$} & \multicolumn{2}{|c|}{$\begin{array}{l}\text { Se evalúan la fiabilidad } \\
\text { de los plazos de entrega }\end{array}$} \\
\hline & $\mathrm{Fa}$ & $\mathrm{Ha}$ & $\mathrm{Fa}$ & $\mathrm{Ha}$ & $\mathrm{Fa}$ & На \\
\hline Siempre(S) & 3 & 15 & 3 & 15 & 7 & 35 \\
\hline Casi Siempre (CS) & 4 & 20 & 11 & 55 & 7 & 35 \\
\hline Algunas Veces (AV) & 10 & 50 & 3 & 15 & 3 & 15 \\
\hline Casi Nunca (CN) & 0 & 0 & 0 & 0 & 0 & 0 \\
\hline $\operatorname{Nunca}(\mathrm{N})$ & 3 & 15 & 3 & 15 & 3 & 15 \\
\hline Total & 20 & 100 & 20 & 100 & 20 & 100 \\
\hline Alternativa & \multicolumn{2}{|c|}{$33 \leq S+C S<66 \%$} & \multicolumn{2}{|c|}{$S+C S \geq 66 \%$} & \multicolumn{2}{|c|}{$S+C S \geq 66 \%$} \\
\hline Tendencia & \multicolumn{2}{|c|}{ Neutral } & \multicolumn{2}{|c|}{ Positiva } & \multicolumn{2}{|c|}{ Positiva } \\
\hline Promedio/Ítemes & \multicolumn{2}{|c|}{3,20} & \multicolumn{2}{|c|}{3,55} & \multicolumn{2}{|c|}{3,75} \\
\hline Categoría & \multicolumn{2}{|c|}{ Moderada presencia } & \multicolumn{2}{|c|}{ Alta presencia } & \multicolumn{2}{|c|}{ Alta presencia } \\
\hline Promedio/Indicador & \multicolumn{6}{|c|}{3,50} \\
\hline Categoría & \multicolumn{6}{|c|}{ Alta presencia } \\
\hline
\end{tabular}

Fuente. Elaboración propia (2019)

A juicio de la investigadora, según los resultados obtenidos se puede inferir que en las empresas de producción social prestadoras de servicio al programa de gerencia petrolera de PDVSA CVP, se adquiere la materia prima o insumos externos lo que es clave para su funcionamiento productivo, garantizando el cumplimiento de las solicitudes realizadas por los clientes.

Lo mostrado, coincide con lo expuesto por Martínez (2006), quien indica que en esta etapa incluye el análisis y evaluación de las ofertas de acuerdo a los criterios establecidos en la etapa de preparación de la compra, por lo que se debe comparar no solamente el precio, sino también entrar en la valoración de factores como la garantía de cumplimiento de especificaciones técnicas, la fiabilidad de los plazos de entrega, la posible continuidad en el suministro, entre otros; repartiendo luego el pedido entre varios proveedores para proteger a la empresa de circunstancias imprevistas. 
Respecto al indicador seguimiento $y$ control, se aprecia en la tabla 5 frecuencias de $85 \%, 70 \%$ y $85 \%$ agrupadas en las opciones siempre y casi siempre, indicando tendencia positiva para todos los ítems. Asimismo, se evidencia un promedio de 3,67, ubicándola en la categoría de alta presencia, afirmando los encuestados que realizan seguimiento al cumplimiento de los plazos acordados con los proveedores para la entrega de los productos comprados, realizan visitas a los proveedores para asegurar el cumplimiento de las condiciones acordadas y verifican el cumplimiento de las especificaciones técnicas de los productos recibidos.

Tabla 5. Indicador: Seguimiento y control

\begin{tabular}{|c|c|c|c|c|c|c|}
\hline \multirow{2}{*}{$\begin{array}{l}\text { Ítemes } \\
\text { Alternativas } \\
\end{array}$} & \multicolumn{2}{|c|}{$\begin{array}{l}\text { Se realiza seguimiento al } \\
\text { cumplimiento de los } \\
\text { plazos acordados con los } \\
\text { proveedores para la } \\
\text { entrega de los productos } \\
\text { comprados. }\end{array}$} & \multicolumn{2}{|c|}{$\begin{array}{l}\text { Realizan visitas a los } \\
\text { proveedores para } \\
\text { asegurar el } \\
\text { cumplimiento de las } \\
\text { condiciones } \\
\text { acordadas. }\end{array}$} & \multicolumn{2}{|c|}{$\begin{array}{l}\text { Se verifica el } \\
\text { cumplimiento de las } \\
\text { especificaciones técnicas } \\
\text { de los productos } \\
\text { recibidos }\end{array}$} \\
\hline & $\mathrm{Fa}$ & На & $\mathrm{Fa}$ & На & $\mathrm{Fa}$ & На \\
\hline Siempre(S) & 7 & 35 & 3 & 15 & 3 & 15 \\
\hline Casi Siempre (CS) & 10 & 50 & 11 & 55 & 14 & 70 \\
\hline Algunas Veces (AV) & 0 & 0 & 0 & 0 & 0 & 0 \\
\hline Casi Nunca (CN) & 0 & 0 & 3 & 15 & 0 & 0 \\
\hline $\operatorname{Nunca}(\mathrm{N})$ & 3 & 15 & 3 & 15 & 3 & 15 \\
\hline Total & 20 & 100 & 20 & 100 & 20 & 100 \\
\hline Alternativa & \multicolumn{2}{|c|}{$S+C S \geq 66 \%$} & \multicolumn{2}{|c|}{$S+C S \geq 66 \%$} & \multicolumn{2}{|c|}{$S+C S \geq 66 \%$} \\
\hline Tendencia & \multicolumn{2}{|c|}{ Positiva } & \multicolumn{2}{|c|}{ Positiva } & \multicolumn{2}{|c|}{ Positiva } \\
\hline Promedio/Ítemes & \multicolumn{2}{|c|}{3,90} & \multicolumn{2}{|c|}{3,40} & \multicolumn{2}{|c|}{3,70} \\
\hline Categoría & \multicolumn{2}{|c|}{ Alta presencia } & \multicolumn{2}{|c|}{ Alta presencia } & \multicolumn{2}{|c|}{ Alta presencia } \\
\hline Promedio/Indicador & \multicolumn{6}{|c|}{3,67} \\
\hline Categoría & \multicolumn{6}{|c|}{ Alta presencia } \\
\hline
\end{tabular}

Fuente. Elaboración propia (2019)

Basado en los resultados descritos anteriormente, a juicio de la investigadora, las empresas de producción social prestadoras de servicio al programa de gerencia petrolera de PDVSA CVP, abordan las sugerencias $y$ problemas de los clientes a fin de fidelizar y crear un mejor lazo con el mismo. Visto así, se valida lo expuesto por Kotler y Armstrong
(2008) quienes plantean que la tarea del especialista en mercadeo no termina con la compra del producto ya que después de adquirido el mismo, el comprador puede quedar satisfecho o no y presentara un comportamiento posterior a la compra la cual es de sumo interés para la empresa. 
En la tabla 6 se resumen los resultados relacionados con la dimensión proceso de compras, con un promedio de 3,36 lo que la ubica en una categoría de moderada presencia, por lo que a juicio de la investigadora las empresas bajo estudio, moderadamente logran adquirir los y gestionar los productos $\mathrm{y} / \mathrm{o}$ servicios necesarios para facilitar el mejoramiento de sus procesos, cubriendo sus necesidades de producción social de manera estratégica con elementos externos a ella, siendo esto un proceso de planificación, organización, dirección y control de actividades.

Tabla 6. Dimensión: Proceso de compras

\begin{tabular}{|c|c|c|c|c|}
\hline \multicolumn{2}{|c|}{ Indicadores } & Promedio & Categoría & Promedio/categoría \\
\hline \multirow{5}{*}{$\begin{array}{c}\text { Proceso de } \\
\text { compras }\end{array}$} & $\begin{array}{l}\text { Detección y } \\
\text { descripción de la } \\
\text { necesidad }\end{array}$ & 2,93 & $\begin{array}{l}\text { Moderada } \\
\text { presencia }\end{array}$ & \\
\hline & $\begin{array}{l}\text { Investigación de } \\
\text { fuentes de } \\
\text { aprovisionamiento }\end{array}$ & 3,05 & $\begin{array}{l}\text { Moderada } \\
\text { presencia }\end{array}$ & 3,36 \\
\hline & $\begin{array}{l}\text { Preparación de la } \\
\text { compra }\end{array}$ & 3,67 & Alta presencia & Moderada presencia \\
\hline & $\begin{array}{l}\text { Ejecución de la } \\
\text { compra }\end{array}$ & 3,50 & Alta presencia & \\
\hline & $\begin{array}{l}\text { Seguimiento y } \\
\text { control }\end{array}$ & 3,67 & Alta presencia & \\
\hline
\end{tabular}

Fuente. Elaboración propia (2019)

Los resultados descritos, permiten validar la teoría expuesta por Hughes (2009), para quien el proceso de compras determina $y$ comunica a través de un sistema de objetivos y políticas mayores, una descripción de lo que se desea que sea la empresa. Los procesos de la gestión de compras, muestran la dirección y el empleo general de recursos y esfuerzos.

CONCLUSIONES

Se describió el proceso de compras en las empresas de producción social prestadoras de servicio a la industria petrolera venezolana, lográndose evidenciar que estas empresas de producción social prestadoras de servicio al programa de gerencia petrolera de PDVSA CVP desarrollan las actividades básicas que les permiten moderadamente detectar y describir las necesidades, investigar fuentes de aprovisionamiento, preparar la compra, ejecutarla de acuerdo a los procedimientos y legislación vigente y realizar el respectivo seguimiento y control. 
No obstante, debido a que no todos los indicadores arrojaron tendencia positiva, se recomienda fortalecer los mecanismos que permitan a los compradores, obtener y analizar periódicamente la información sobre los materiales que más rotan en los almacenes, antes de iniciar la compra de un grupo de bienes. Al mismo tiempo continuar o iniciar proyectos de investigación de mercados, que hagan posible obtener información actualizada sobre las características de los mercados en los que se demandan los productos más críticos.

Mantener el establecimiento de contratos - alianzas para las compras de carácter estratégico en la empresa, dado que esta actividad garantiza la continuidad de suministro, permite crear relaciones estrechas con los proveedores importantes y evita el retrabajo que pueden causar las compras menores recurrentes. Finalmente asegurar el cumplimiento de condiciones acordadas con los proveedores, mediante el diseño y ejecución de programas de auditorías o visitas, para los bienes que lo ameriten por su impacto operacional.

\section{REFERENCIAS}

Heredia, N. (2007). Gerencia de compras: La nueva estrategia competitiva. Primera Edición. Edición Ltda. Colombia

Martínez, E. (2007). Gestión de compras: Negociación y estrategias de aprovisionamiento. Cuarta Edición. FC Editorial. España

Vásquez, N. (2008). Organización en el Nuevo Modelo Social. Caracas-Venezuela.

PDVSA página oficial de internet (2019). Disponible en: http://www.pdvsa.com/index.php?tpl=interf ace.sp/design/readmenuprinc_eps.tpl.html \&newsid_temas $=220 \quad$ Consultdo el : 02/08/2019

Kotler, P. y Armstrong, G. (2008). Fundamentos de marketing. Pearson Educación. México.

Hawkins, D. Best, R. y Coney, K. (2004). Comportamiento del consumidor: Construyendo estrategias de marketing. McGraw-Hill Interamericana. México.

Arellano, R. (2002). Comportamiento del consumidor y Marketing: Aplicaciones para América Latina. Editorial Harla. México

Montoya, A. (2002). Conceptos modernos de administración de compras. Editorial Norma, S.A. Colombia 